\title{
A LIGHT-FRONT QUARK MODEL FOR THE ELECTROMAGNETIC FORM FACTOR OF THE PION
}

\author{
J. P. B. C. DE MELO \\ IFT, Universidade Estadual Paulista, São Paulo, SP, Brazil \\ T. FREDERICO \\ Dep. de Física, ITA, CTA, São José dos Campos, São Paulo, Brazil \\ E. PACE \\ Dipartimento di Fisica, Università di Roma "Tor Vergata" and Istituto \\ Nazionale di Fisica Nucleare, Sezione Tor Vergata, Via della Ricerca Scientifica \\ 1, I-00133 Roma, Italy \\ G. SALMÈ \\ Istituto Nazionale di Fisica Nucleare, Sezione Roma I, P.le A. Moro 2, I-00185 \\ Roma, Italy
}

\begin{abstract}
In this contribution, an approach for a unified description of the pion electromagnetic form factor, in the space- and time-like regions, within a constituent quark model on the light front, will be reviewed. Our approach is based on i) the onshell quark-hadron vertex functions in the valence sector, ii) the dressed photon vertex where a photon decays in a quark-antiquark pair, and iii) the emission and absorption amplitudes of a pion by a quark. Results favorably compare with the existing experimental data.
\end{abstract}

\section{Introduction}

Constituent quark models (CQM) developed within a light-front framework (for a general review, see, e.g., Ref. [1]) appear to be an interesting tool for investigating the electromagnetic properties of hadrons. One of the main advantage of the light-front approach is that the Fock vacuum (i.e. the vacuum of a free theory) could represent a reliable approximation of the physical, interacting vacuum, given the positivity constraint and the kinematical nature of the plus component of the total momentum, $P^{+}$. Then, expanding hadron states over a Fock basis becomes meaningful, and 
one can safely write for a meson

$$
\begin{aligned}
& \left.\mid \text { meson; } P^{+} \mathbf{P}_{\perp} ; \zeta\right\rangle=\prod_{i=1,2} \int d \mathbf{k}_{i \perp} d \xi_{i} \delta\left(\sum_{i=1,2} \mathbf{k}_{i \perp}\right) \delta\left(1-\sum_{i=1,2} \xi_{i}\right) \times \\
& \sum_{\left\{\zeta_{j}\right\}} \psi_{q \bar{q}}\left(\mathbf{k}_{i \perp}, \xi_{i}, \zeta_{j}\right)\left|q \bar{q} ; P^{+} \mathbf{P}_{\perp} ; \mathbf{k}_{i \perp}, \xi_{i}, \zeta_{j}\right\rangle+ \\
& +\prod_{i=1,4} \int d \mathbf{k}_{i \perp} d \xi_{i} \delta\left(\sum_{i=1,4} \mathbf{k}_{i \perp}\right) \delta\left(1-\sum_{i=1,4} \xi_{i}\right) \times \\
& \sum_{\left\{\zeta_{j}\right\}} \psi_{q \bar{q} q \bar{q}}\left(\mathbf{k}_{i \perp}, \xi_{i}, \zeta_{j}\right)\left|q \bar{q} q \bar{q} ; P^{+} \mathbf{P}_{\perp} ; \mathbf{k}_{i \perp}, \xi_{i}, \zeta_{j}\right\rangle+ \\
& +\prod_{i=1,3} \int d \mathbf{k}_{i \perp} d \xi_{i} \delta\left(\sum_{i=1,3} \mathbf{k}_{i \perp}\right) \delta\left(1-\sum_{i=1,3} \xi_{i}\right) \times \\
& \sum_{\left\{\zeta_{j}\right\}} \psi_{q \bar{q} g}\left(\mathbf{k}_{i \perp}, \xi_{i}, \zeta_{j}\right)\left|q \bar{q} g ; P^{+} \mathbf{P}_{\perp} ; \mathbf{k}_{i \perp}, \xi_{i}, \zeta_{j}\right\rangle+\ldots . .
\end{aligned}
$$

where a light-front spin component along the $z$-axis is indicated by $\zeta$ and the function $\psi$ 's are the intrinsic amplitudes for the corresponding Fock states. The possibility to include, in a given framework, states beyond the familiar CQM ones (i.e. $|q \bar{q}\rangle$ for the mesons and $|q q q\rangle$ for the baryons) allows one to address the rich phenomenology in the time-like (TL) region within a unified approach, and, in turn, to increase the constraints to be fulfilled by a chosen model.

Our model ${ }^{2}$ has been developed for investigating the electromagnetic form factor of a pion in the whole range of momentum transfer, i.e. for positive and negative squared mass of the virtual photon. The first building block is represented by the Mandelstam formula for the matrix elements of the em current ${ }^{3}$. Following Ref. 3 , the matrix elements for the pion in the TL region read

$$
\begin{aligned}
& j^{\mu}=e \frac{2 m^{2}}{\imath f_{\pi}^{2}} N_{c} \int \frac{d^{4} k}{(2 \pi)^{4}} \bar{\Lambda}_{\pi}\left(k, P_{\pi}\right) \Lambda_{\bar{\pi}}\left(k-P_{\pi}, P_{\bar{\pi}}\right) \times \\
& \operatorname{Tr}\left[S\left(k-P_{\pi}\right) \gamma^{5} S(k-q) \Gamma^{\mu} S(k) \gamma^{5}\right]
\end{aligned}
$$

where $S(p)=[\not p-m+\imath \epsilon]^{-1}$, with $m$ the mass of the constituent quark, $\Gamma^{\mu}(k, q)$ is the quark-photon vertex, $q^{\mu}$ the virtual-photon momentum, $\Lambda_{\pi}\left(k, P_{\pi}\right)$ the pion vertex function, $P_{\pi}^{\mu}$ and $P_{\bar{\pi}}^{\mu}$ are the pion and antipion momenta, respectively. $N_{c}=3$ is the number of colors and the factor 2 comes from the isospin weight, since we are dealing with a charged pion 
form factor. For the space-like (SL) region, $P_{\pi}^{\mu}$ has to be replaced by $-P_{\pi}^{\mu}$ and $\bar{\pi}$ by $\pi^{\prime}$.

A key mathematical step is given by the four dimensional integration of an integrand that has, in principle, a very complicated analytical structure, due to the presence of poles from the fermion propagators and from the analytical structure of the vertex functions. A first approximation is introduced as one projects out the Mandelstam formula on the light front by a $k^{-}$integration (see Figs 1 and 2). In particular, we have assumed that: i) the meson vertex functions do not diverge in the complex plane $k^{-}$for $\left|k^{-}\right| \rightarrow \infty$ and ii) the contributions of their singularities are negligible. To emphasize the unified description of the em form factor in TL and SL regions, the simplifying assumption of a chiral pion $\left(m_{\pi}=0\right)$ has been adopted. As a matter of fact, in the SL case we have carried out our analysis in a frame where $\mathbf{P}_{\pi \perp}=\mathbf{P}_{\pi / \perp}=\mathbf{0}$, obtaining that $P_{\pi}^{+}=q^{+}\left(-1+\sqrt{1-4 m_{\pi}^{2} / q^{2}}\right) / 2$. In the limit $m_{\pi} \rightarrow 0$ one has $P_{\pi}^{+}=0$ and $P_{\pi \prime}^{+}=q^{+}$. Then, only the contribution of the pair-production mechanism survives, see Fig. 1(b).

In the TL case, the choice $\mathbf{P}_{\bar{\pi} \perp}=-\mathbf{P}_{\pi \perp}=\mathbf{0}$ leads to $P_{\pi}^{+}=q^{+}(1 \pm$ $\left.\sqrt{1-4 m_{\pi}^{2} / q^{2}}\right) / 2$. In the limit $m_{\pi} \rightarrow 0$, in analogy with the SL case, we have adopted the choice $P_{\pi}^{+}=0$. Then, only the contribution of the diagram (b) in Fig. 2 survives. To complete our model $^{2}$ we have to answer the following

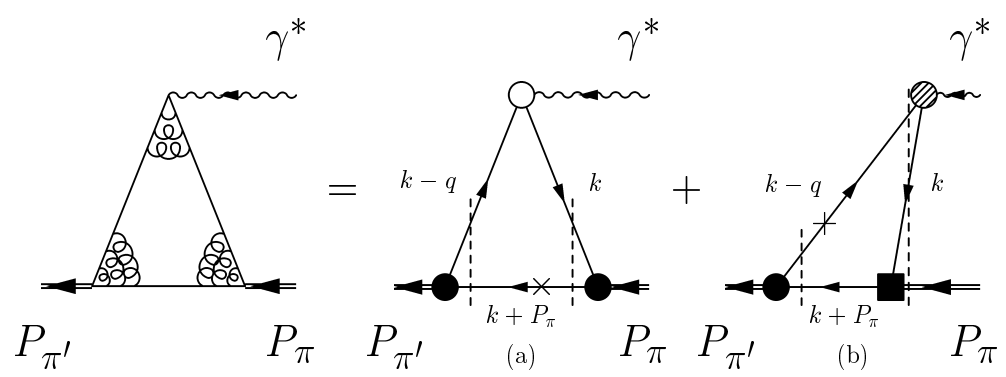

Figure 1. Light-front time-ordered diagrams contributing to the em form factor of the pion in the space-like region. In contribution $(a)$ one has $0<k^{+}+P_{\pi}^{+}<P_{\pi}^{+}$, while in (b) $0<k^{+}<q^{+}$. Vertical dashed lines indicate a fixed value for the light-front time $x^{+}$ that flows from the right hand toward the left hand.

After Ref. [2]

questions : i) how to describe the $q \bar{q}$-meson vertices ? ii) how to model the dressed quark-photon vertex ? iii) how to deal with the amplitude for the emission or absorption of a pion by a quark ? In the following section, our 


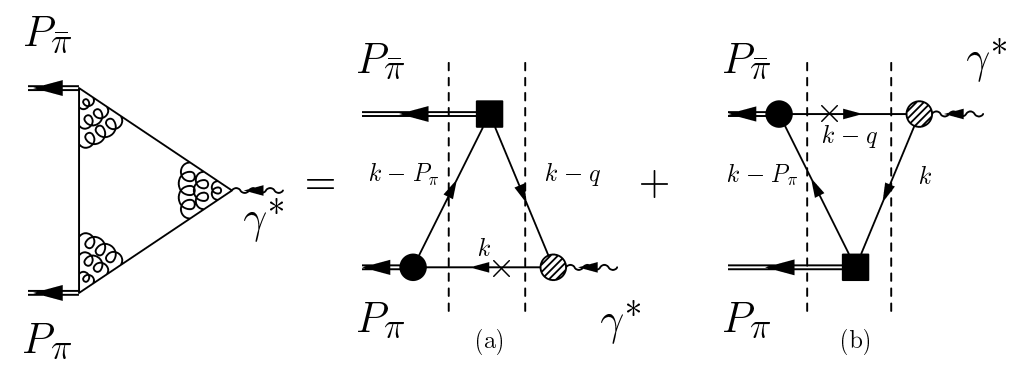

Figure 2. Light-front time-ordered diagrams contributing to the em form factor of the pion in the time-like region. In contribution $(a)$ one has $0<k^{+}<P_{\pi}^{+}$, while in (b) $P_{\pi}^{+}<k^{+}<q^{+}$.

After Ref. [2]

answers will be illustrated in some detail.

\section{The Model}

In order to construct our model, we have first introduced an approximation for the momentum component of the pion vertex function, $\Lambda_{\pi}\left(k, P_{\pi}\right)$, when both the quarks are on their mass shell and in the interval $0 \leq k^{+} \leq P_{\pi}^{+}$. Such a function of the quark light-front momenta, can be approximated by the "light-front pion wave function", $\psi_{\pi}$, obtained within the Hamiltonian light-front dynamics, through the following equation

$$
\begin{aligned}
& \left(\not k_{o n}+m\right) \gamma^{5} \psi_{\pi}\left(k^{+}, \mathbf{k}_{\perp} ; P_{\pi}^{+}, \mathbf{P}_{\pi \perp}\right)\left[\left(\not k-P_{\pi}\right)_{o n}+m\right]= \\
& \left(\not k_{o n}+m\right) \gamma^{5} \frac{m}{f_{\pi}} \frac{P_{\pi}^{+}}{\left[m_{\pi}^{2}-M_{0}^{2}\left(k^{+}, \mathbf{k}_{\perp} ; P_{\pi}^{+}, \mathbf{P}_{\pi \perp}\right)\right]}\left[\Lambda_{\pi}\left(k, P_{\pi}\right)\right]_{\left[k^{-}=k_{o n}^{-}\right]} \times \\
& {\left[\left(k-P_{\pi}\right)_{o n}+m\right]}
\end{aligned}
$$

where $k_{o n}^{-}=\left(\mathbf{k}_{\perp}^{2}+m^{2}\right) / k^{+}$and $M_{0}$ is the light-front free mass (see, e.g., Ref. [4]). A similar relation is also adopted for the vector mesons (VM). For the pion and the VM wave functions, we have used the eigenfunctions of the square mass operator proposed in Refs. [5, 6], within a relativistic constituent quark model which achieves a natural explanation of the "Iachello-Anisovitch law" 7,8. The VM eigenfunctions,$\psi_{n}\left(k^{+}, \mathbf{k}_{\perp} ; q^{+}, \mathbf{q}_{\perp}\right)$, are normalized to the probability of the lowest $(q \bar{q})$ Fock state, roughly estimated to be $\sim 1 / \sqrt{2 n+3 / 2}$ in a simple model ${ }^{2}$ that reproduces the "Iachello-Anisovitch law" 7,8 ( $n$ is the principal quantum number).

As for the second question, following Ref. [2], a Vector Meson Dominance (VMD) approximation is applied to the quark-photon vertex $\Gamma^{\mu}(k, q)$, 
when a $q \bar{q}$ pair is produced. In particular, the plus component of the quarkphoton vertex reads as follows (see Fig. 3)

$$
\begin{aligned}
& \Gamma^{+}(k, q)=\sum_{n, \lambda}\left[\epsilon_{\lambda} \cdot \widehat{V}_{n}(k, k-q)\right] \Lambda_{n}\left(k, P_{n}\right) \times \\
& \frac{\sqrt{2}\left[\epsilon_{\lambda}^{+}\right]^{*} f_{V n}}{\left(q^{2}-M_{n}^{2}+\imath M_{n} \Gamma_{n}\left(q^{2}\right)\right)}
\end{aligned}
$$

where $f_{V n}$ is the decay constant of the n-th vector meson into a virtual photon, $M_{n}\left(P_{n}\right)$ the mass (four-momentum) of the VM, $\Gamma_{n}\left(q^{2}\right)=\Gamma_{n} q^{2} / M_{n}^{2}$ (for $q^{2}>0$ ) the corresponding total decay width, $\epsilon_{\lambda}$ the VM polarization, and $\left[\epsilon_{\lambda} \cdot \widehat{V}_{n}(k, k-q) \Lambda_{n}(k, q)\right.$ the VM vertex function ( for the VM Dirac structure, $\widehat{V}_{n}$, see below). The decay constant, $f_{V n}$, is evaluated starting

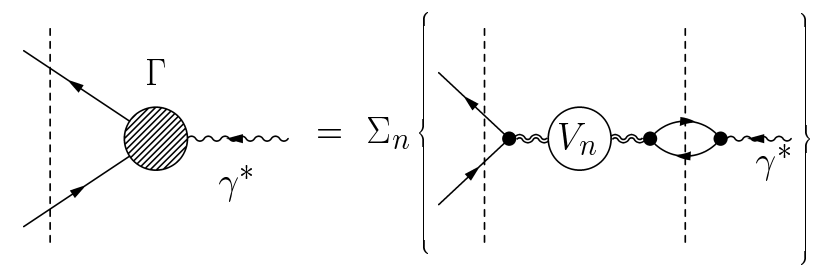

Figure 3. Diagrammatic analysis of the quark-photon vertex, $\Gamma^{\mu}(k, q)$. After Ref. [2]

from a four dimensional representation in terms of the VM Bethe-Salpeter vertex and integrating over $k^{-}$. In particular, one has

$$
\begin{aligned}
& f_{V n}=-\imath \frac{N_{c}}{4(2 \pi)^{4}} \int d k^{-} d k^{+} d \mathbf{k}_{\perp} \frac{\operatorname{Tr}\left[\gamma^{+} \mathcal{V}_{n z}\left(k, k-P_{n}\right)\right] \Lambda_{n}\left(k, P_{n}\right)}{\left[k^{2}-m^{2}+\imath \epsilon\right]\left[\left(P_{n}-k\right)^{2}-m^{2}+\imath \epsilon\right]}= \\
& =-\frac{N_{c}}{4(2 \pi)^{3}} \int_{0}^{P_{n}^{+}} d k^{+} d \mathbf{k}_{\perp} \frac{\operatorname{Tr}\left[\gamma^{+} \mathcal{V}_{n z}\left(k, k-P_{n}\right)\right]}{k^{+}\left(P_{n}^{+}-k^{+}\right)} \psi_{n}\left(k^{+}, \mathbf{k}_{\perp} ; M_{n}, \overrightarrow{0}_{\perp}\right)(5
\end{aligned}
$$

where $\mathcal{V}_{n z}\left(k, k-P_{n}\right)=\left(\not k-P_{n}+m\right) \widehat{V}_{n z}\left(k, k-P_{n}\right)(\not k+m)$.

Note that only ${ }^{3} S_{1}$ isovector vector mesons have been taken into account in the calculations, and therefore the on-mass-shell spinorial part of the VM vertex must reproduce the well-known Melosh rotations for ${ }^{3} S_{1}$ states, (see, e.g., Ref. [4]), viz.

$$
\widehat{V}_{n}^{\mu}(k, k-q)=\gamma^{\mu}-\frac{k_{o n}^{\mu}-(q-k)_{o n}^{\mu}}{M_{0}\left(k^{+}, \mathbf{k}_{\perp} ; q^{+}, \mathbf{q}_{\perp}\right)+2 m} .
$$

The third question raised in the Introduction is answered by describing with a constant ${ }^{9}$ the amplitude for the emission or absorption of a pion 
by a quark, i.e. the pion vertex function in the non-valence sector. The value of the constant is fixed by the pion charge normalization, given our simplifying assumption of a chiral pion.

In the limit $m_{\pi} \rightarrow 0$ the pion form factor receives contributions only from processes where the photon decays in a $q \bar{q}$ pair. Then, by means of Eq. (4) the matrix element $j^{+}$can be written as a sum over the vector mesons, and consequently the form factor becomes

$$
F_{\pi}\left(q^{2}\right)=\sum_{n} \frac{f_{V n}}{q^{2}-M_{n}^{2}+\imath M_{n} \Gamma_{n}\left(q^{2}\right)} g_{V n}^{+}\left(q^{2}\right)
$$

where $g_{V n}^{+}\left(q^{2}\right)$, for $q^{2}>0$, is the form factor for the VM decay in a pair of pions.

Each VM contribution to the sum (7) is invariant under kinematical light-front boosts and can be evaluated in the rest frame of the corresponding resonance (with $q^{+}=M_{n}$ and $\mathbf{q}_{\perp}=0$ ).

It turns out that the same expression for $g_{V n}^{+}\left(q^{2}\right)$ holds both in the TL and in the SL regions ${ }^{2}$.

\section{Results}

In our calculations the up-down quark mass is fixed at $0.265 \mathrm{GeV}^{6}$ and the oscillator strength at $\omega=1.39 \mathrm{GeV}^{2}{ }^{8}$. For the first four vector mesons the known experimental masses and widths are used ${ }^{10}$ (see Table I), a part the value of the mass of $\rho$-meson changed to $m_{\rho}=0.750 \mathrm{Gev}$, in order to reproduce the correct position of the $\rho$ peak in the time-like form factor of the charged pion. For the VM with $M_{n}>2.150 \mathrm{GeV}$, the mass values corresponding to the model of Ref. 6 are used, while for the unknown widths we use a single value $\Gamma_{n}=0.15 \mathrm{GeV}$. To obtain stability of the results up to $q^{2}=10(\mathrm{GeV} / \mathrm{c})^{2}$ twenty resonances are considered.

The calculated pion form factor is shown in Fig. 4 in a wide region of square momentum transfers, from $-10(\mathrm{GeV} / \mathrm{c})^{2}$ up to $10(\mathrm{GeV} / \mathrm{c})^{2}$. The VM dominance ansatz for the (dressed photon )- $(q \bar{q})$ vertex, within a CQ model consistent with the meson spectrum, is able to give a unified description of the pion form factor both in the SL and TL regions.

The SL form factor is notably well described, see Fig. 5, from the high$q^{2}$ region to the low- $q^{2}$ one, as well as the charge radius. Finally, it is worth noting that the heights of the TL bumps directly depend on the calculated values of $f_{V n}$ and $g_{V n}^{+}$, and for the sake of completeness a table of calculated em decay widths, $\Gamma_{e^{+} e^{-}}^{t h}$, are shown in Table II. 
Table I. Input values of our model, $M_{n}(P D G)$ and $\Gamma_{n}(P D G)$ (see text). For the sake of comparison, the masses evaluated in the model of Refs. [5, 6], $M_{n}(\mathrm{FPZ})$ are also shown (the mass of the $\rho$ is an input for this model).

\begin{tabular}{|c||c|c||c|}
\hline $\mathrm{VM}$ & $M_{n}(\mathrm{FPZ})$ & $M_{n}(P D G)$ & $\Gamma_{n}(P D G)$ \\
\hline$\rho(770)$ & $.770 \mathrm{GeV}$ & $.770 \mathrm{GeV}$ & $0.15 \mathrm{GeV}$ \\
\hline$\rho(1450)$ & $1.408 \mathrm{GeV}$ & $1.465 \mathrm{GeV}$ & $0.37 \mathrm{GeV}$ \\
\hline$\rho(1770)$ & $1.836 \mathrm{GeV}$ & $1.723 \mathrm{GeV}$ & $0.30 \mathrm{GeV}$ \\
\hline$\rho(2150)$ & $2.182 \mathrm{GeV}$ & $2.149 \mathrm{GeV}$ & $0.18 \mathrm{GeV}$ \\
\hline
\end{tabular}

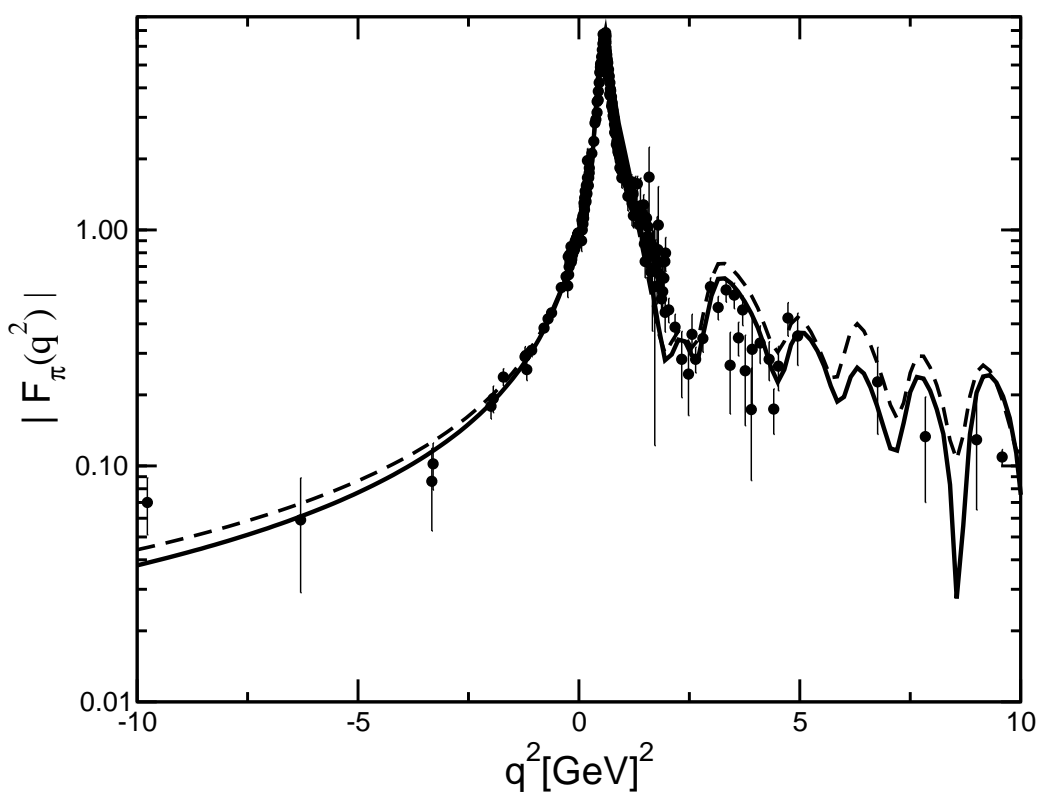

Figure 4. Pion electromagnetic form factor vs. the square momentum transfer $q^{2}$. Solid line: result obtained by using the full pion wave function. Dashed line: result obtained by using the asymptotic pion wave function (After Ref. 2).

\section{Perspectives}

The results obtained within our approach ${ }^{2}$ encourage an investigation of the TL form factors of the nucleon based on a simple ansatz for the nonvalence component of the nucleon state, following as a guideline the pion 

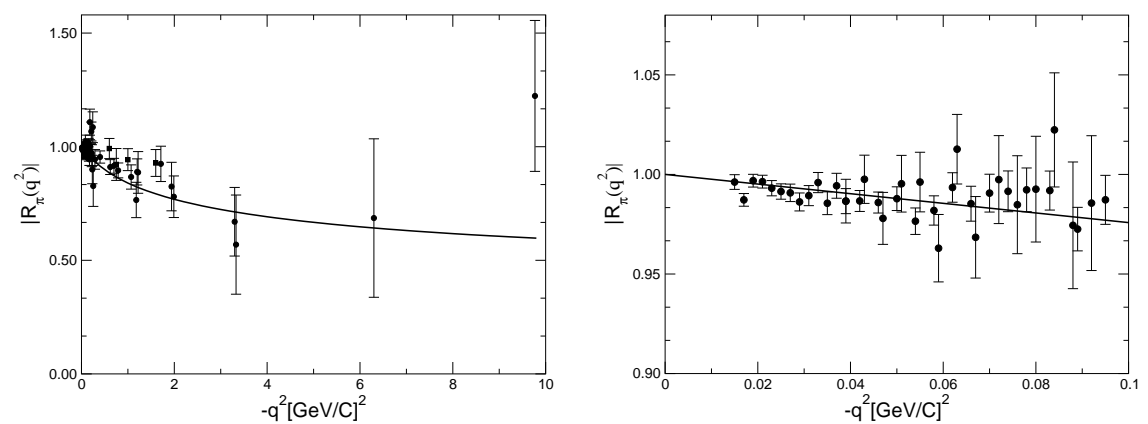

Figure 5. The ratio $R_{\pi}\left(q^{2}\right)=F_{\pi}\left(q^{2}\right) /\left[1 /\left(1-q^{2} / m_{\rho}^{2}\right)\right]$ vs $q^{2}$, in the SL region $\left(m_{\rho}=0.770 \mathrm{GeV}\right)$. The rightmost figure shows in great detail the region $0 \leq-$ $q^{2} \leq 0.1(\mathrm{GeV} / \mathrm{c})^{2}$, relevant for evaluating the charge radius.

Experimental data from R. Baldini et al. ${ }^{11}$

Table II. Em decay widths, $\Gamma_{e^{+} e^{-}}^{t h}$, for the first three $\rho$-mesons

\begin{tabular}{|c||c|c|}
\hline $\mathrm{VM}$ & $\Gamma_{e^{+} e^{-}}^{t h}$ & $\Gamma_{e^{+} e^{-}}^{\exp }$ \\
\hline$\rho(770)$ & $6.37 \mathrm{KeV}$ & $6.77 \pm 0.32 \mathrm{KeV}$ \\
\hline$\rho(1450)$ & $1.61 \mathrm{KeV}$ & $>2.30 \pm 0.50 \mathrm{KeV}$ \\
\hline$\rho(1770)$ & $1.23 \mathrm{KeV}$ & $>0.18 \pm 0.10 \mathrm{KeV}$ \\
\hline
\end{tabular}

case.

\section{References}

1. S J. Brodsky, H.C. Pauli, and S.S. Pinsky, Phys. Rep. 301, 299 (1998).

2. J.P.B.C. de Melo, T. Frederico, E. Pace and G. Salmè, Phys. Lett. B581,75 (2004) and to be published.

3. S. Mandelstan, Proc. Royal Soc. (London) A233, 248 (1956).

4. W. Jaus, Phys. Rev. D41, 3394 (1990).

5. T. Frederico and H.-C. Pauli, Phys. Rev. D64, 054007(2001).

6. T. Frederico, H.-C. Pauli and S.-G. Zhou, Phys. Rev. D66, 054007 (2002); ibidem D66, 116011 (2002).

7. F. Iachello, N.C. Mukhopadhyay and L. Zhang, Phys. Rev. D44, 898 (1991).

8. A.V. Anisovitch, V.V. Anisovich, and A.V. Sarantsev, Phys. Rev. D62, 051502(R) (2000).

9. C.-R. Ji and H.-M. Choi, Phys. Lett. B513, 330 (2001).

10. K. Hagiwara et al., Phys. Rev. D66, 010001 (2002).

11. R. Baldini, et al., Eur. Phys. J. C11, 709 (1999); Nucl. Phys. A666\&667, 3 (2000) and private communication. 